\title{
Una historia con olor a leche: de la desnutrición a la obe- sidad, políticas públicas e ideologías de género'
}

Isabel Pemjean ${ }^{2}$

\begin{abstract}
Resumen
El objetivo de este artículo es presentar los principales resultados de un estudio que buscó conocer el desarrollo de las políticas públicas nutricionales del Estado chileno, desde inicios del siglo XX hasta la actualidad, enfatizando en la injerencia de estas políticas en la construcción de los roles de género en Chile. En este sentido, desde una mirada cronológica se reconoce un estrecho vínculo entre malnutrición, mortalidad infantil y constitución política de la salud como necesidad pública, relación que deja una huella importante no sólo en los modos de alimentarse de la población, sino también en sus ideologías de género.
\end{abstract}

Palabras clave: género - salud - políticas nutricionales - siglo XX.

\begin{abstract}
The aim of this article is to present the main results of a study that sought to know the development of the public nutritional policies of the chilean State, from the beginning of the 20th century until present days, emphasizing the interference of these policies in the construction of gender roles in Chile. In this respect, from a chronological look, it's recognized a close link between malnutrition, infant mortality and health policy constitution as a public necessity, relationship that leaves an important mark not only in ways the population feeds themselves, but also in their gender ideologies.
\end{abstract}

Key words: gender - health - nutrition policies $-20^{\text {th }}$ century.

El artículo es el resultado de una investigación realizada en el marco del Proyecto Anillo de Estudios Interdisciplinarios de Género y Cultura, 2008-2011, CONICYT. La información presentada es fruto tanto de entrevistas como de la revisión de fuentes de segundo orden.

2 Antropóloga por la Universidad de Chile. Magíster en Estudios de Género y Cultura, mención Ciencias Sociales por el Centro Interdisciplinario de Estudios de Género (CIEG) de la misma Universidad. Trabaja en el CIEG y es académica del Departamento de Antropología de la Universidad de Chile. Participa como investigadora joven en el Anillo de Estudios Interdisciplinarios de Género y Cultura, 2008-2011, CONICYT. 


\section{INTRODUCCIÓN}

En toda la gama de las acciones humanas es difícil encontrar algo más central y cotidiano que la alimentación. Estamos obligados/as a comer, tanto para la supervivencia física y el bienestar síquico como para la reproducción social de las sociedades humanas. Pero, por más cotidiano que sea alimentarse, parece ser uno de los grandes temas de nuestras sociedades. Nos referimos a él sin cesar, preguntándonos sobre lo bueno y lo malo para comer en nuestras conversaciones cotidianas, en la prensa, la literatura, la publicidad y la medicina. El qué comer se vuelve una situación a resolver, pues debemos tomar decisiones y hacer elecciones desde las cuales dictamos lo correcto y lo incorrecto, lo moral y lo amoral, las normas que rigen nuestros comportamientos alimentarios.

Se trata de ideologías nutricionales que, más o menos marcadas, están presentes en cada uno/a de nosotros/as, en las que juegan, entre otras, normas familiares en tanto valores, creencias y prácticas alimenticias que nos han sido heredadas; las religiosas; las biológicas y las médicas, referidas a las normas y recomendaciones sobre lo que es una comida adecuada tanto cualitativa como cuantitativamente, basadas en conocimientos científico-nutricionales.

Ahora bien, para el presente artículo nos centraremos en la injerencia de la salud en los discursos alimentarios de la población, preguntándonos específicamente por la correlación entre las políticas sociales de salud y las ideologías nutricionales de la población.

Para contestar estas interrogantes se vuelve necesario mirar cronológicamente la constitución de la medicina social en Chile, en la que iremos constatando un estrecho vínculo entre desnutrición, mortalidad infantil y constitución política de la salud como necesidad pública; además de las marcas que irá dejando no sólo en los modos de alimentarse de la población, sino también en sus ideologías de género.

Durante el siglo XX, Chile recorrió la ruta de la malnutrición³. Si bien los problemas de desnutrición se inician antes de 1900, esta es la fecha escogida para iniciar el marco temporal de este relato, principalmente por ser el año en que se implementa, por primera vez en Chile, una medida importante en torno a la malnutrición infantil: las llamadas Gotas de Leche, a las que se hará referencia más adelante. De esta manera, se puede considerar el período de 1900 a 1990 como el de desnutrición y alta mortalidad infantil en nuestro país. Luego, la década del '90 se plantea como una fase de

La malnutrición es la consecuencia de no cumplir con una dieta equilibrada en calidad y en cantidad. Puede ocurrir por exceso y llevar a obesidad, o por defecto, llevando a desnutrición. 
transición en la que comienzan a coexistir los tipos de malnutrición por exceso y por defecto, ganando la primera desde el año 2000 en adelante.

Pero este fenómeno no es para nada único, por el contrario, la transición nutricional o la"secuencia de características y cambios del estado nutricional, que resultan como consecuencia de los cambios en la estructura general de la dieta correlacionada con cambios económicos, demográficos, sociales y de salud" (Albala et al., 2000: s/p) ha sido definida como parte del camino al desarrollo de un país. Siguiendo el modelo europeo, hay cuatro etapas: i) una de pre transición nutricional, caracterizada por una dieta escasa en grasas y azúcares, donde predomina la desnutrición; ii) una de transición donde dichos alimentos aumentan, generando una población en la que quienes no son desnutridos/ as son obesos/as; iii) una tercera etapa en que las grasas y azúcares se mantienen, predominando la obesidad; y iv) una última en que se produce una combinación y equilibrio de las dos primeras, reduciendo la malnutrición por exceso.

Claramente, estos procesos se insertan en contextos mayores en los que influye con fuerza la situación económica, doblándose la obesidad donde el ingreso per cápita supera un crecimiento del 7\%, pues, lamentablemente, y sobre todo en América Latina, el tener mayor acceso a los alimentos no asegura su calidad. A esto se suman las acciones implementadas desde la salud pública en cuanto a higiene y sanidad, particularmente en lo relacionado con servicios básicos asegurados para toda la población, y en cuanto a la definición de pautas nutricionales que normen lo bueno y lo malo para comer.

\section{Tabla 1 FASES DE TRANSICIÓN NUTRICIONAL}

\begin{tabular}{|c|c|c|c|c|}
\hline & $\begin{array}{l}\text { 1a etapa: } \\
\text { Pre }\end{array}$ & 2a etapa: Transición & $\begin{array}{l}\text { 3a etapa: } \\
\text { Post }\end{array}$ & $4^{a}$ etapa \\
\hline Dieta & $\begin{array}{l}\text { Cereales } \\
\text { Vegetales } \\
\text { Frutas } \\
\text { Tubérculos } \\
\text { (sin mucha variación) }\end{array}$ & $\begin{array}{l}\text { Aumento azúcar, grasas } \\
\text { y alimentos procesados. }\end{array}$ & $\begin{array}{l}\text { Contenido alto de grasa } \\
\text { y azúcar. Contenido bajo } \\
\text { en fibra. }\end{array}$ & $\begin{array}{l}\text { Menor cantidad de } \\
\text { grasas y alimentos } \\
\text { procesados. Aumento } \\
\text { de frutas, vegetales, } \\
\text { lácteos descremados y } \\
\text { cereales. }\end{array}$ \\
\hline Estado nutricional & $\begin{array}{l}\text { Predominan deficiencias } \\
\text { nutricionales }\end{array}$ & $\begin{array}{l}\text { Coexisten deficiencias } \\
\text { nutricionales por déficit } \\
\text { y exceso. }\end{array}$ & $\begin{array}{l}\text { Predominio de obesidad } \\
\text { e hiperlipidemias. }\end{array}$ & $\begin{array}{l}\text { Reducción de obesidad } \\
\text { e hiperlipidemias y } \\
\text { mejoría ósea. }\end{array}$ \\
\hline
\end{tabular}

Fuente: Illanes (1993)

Nuestro país es, actualmente, uno de los pocos en América Latina insertos en la tercera fase. La mayoría está en la segunda, lo que significa que, a diferencia de Chile, estos países no han logrado disminuir cifras críticas de desnutrición. Pero, ¿cuándo se logra superar en nuestro país la malnutrición por déficit? ¿Mediante qué políticas? ¿En qué años se crea el Ministerio de Salud (MINSAL) con su consecuente rol social? 
¿Cuáles han sido las líneas más fuertes en temas nutricionales de la salud pública?, y, ¿de qué manera han influido en los discursos alimentarios de la población?

A continuación se entrega una mirada sobre lo que han sido las políticas nutricionales en Chile desde 1900 en adelante. Ahora bien, teniendo en cuenta que, a nivel local, el siglo XX marcó la construcción del Estado y la nación, se escogió una presentación cronológica capaz de dar cuenta de la coincidencia de los distintos enfoques y momentos de las medidas públicas nutricionales con los cambios en las grandes tendencias gubernamentales. Ello pues, desde nuestra visión, dicha correspondencia demuestra la relevancia de las políticas públicas, y en especial de las asociadas a la salud, en la política y el Estado chileno independiente.

\section{MODERNIDAD Y LUCHA CONTRA LA MORTALIDAD INFANTIL (1900-1990)}

La desnutrición se siente con fuerza en Chile desde el año 1900 y se extiende hasta la década del '90, manteniendo los niveles más altos de mortalidad infantil a nivel mundial. Es un período que converge con fenómenos sociales, políticos y económicos fundamentales en la historia de nuestro país. Se trata de una época de ingreso y fortalecimiento de la modernidad, en que población y dirigentes intentan mejorar las condiciones de vida generales. Son las décadas de la expansión de la educación, del derecho a voto de las mujeres, de la formación de los cuadros políticos, de las grandes transformaciones culturales y, también, de la creación de un sistema público de salud. Son noventa años que no pasarán desapercibidos, entre los que es posible identificar tres grandes momentos: i) el Estado liberal, como los años en que la desnutrición infantil se vuelve crítica, en especial por la rápida migración rural-urbana y el crecimiento ilimitado de los conventillos en la ciudad; ii) el Estado desarrollista-liberal, como la constatación de la necesidad de institucionalizar este problema en la agenda pública; y iii) la Dictadura como la superación del problema.

\section{La mortalidad infantil más alta del mundo (Estado liberal, 1900-1938)}

Si bien el Servicio Nacional de Salud (SNS), antecesor del MINSAL, no se crea hasta 1952, es necesario rastrear los orígenes de la salud pública y en especial de las medidas nutricionales, desde inicios del siglo XX, cuando la sociedad chilena vive la llamada "cuestión social". Se produce una situación de crisis en que la gran mayoría de la población vivía en pésimas condiciones, hacinada en conventillos, en deplorables situaciones higiénicas detonantes de pestes (viruela, cólera, alfombrilla) y de enfermedades infecciosas (tuberculosis, fiebre tifoidea), causantes de una alta mortalidad infantil. La más alta del mundo, por cierto.

"Vivimos en dos piezas de una pobre hilera de piezas que son una especie de conventillo con patio compartido. Las puertas de las piezas se suceden una casi al lado de la otra y miran a un ancho espacio, al frente en la esquina hay una casa grande, después más casas o simplemente un cerco. Es el otro límite del ancho 
callejón. El fondo ciego del callejón es un gran portón de trancas de madera siempre cerrado, que es la entrada a un campo" (Hernández, 2008: s/p).

Se trataba de obreros/as, migrantes rurales que, luego del paso al sistema fabril, vinieron a la ciudad en busca de empleo. En esta época, las mujeres y los/as niños/as representaban un tercio del total de la fuerza laboral, al igual que el ingreso aportado a la familia. Hambre, cesantía, prostitución, hacinamiento, insalubridad, explotación, abandono y criminalidad: la primera mitad del siglo XX marcó el paso de la crisis de la oligarquía a la modernidad populista.

A una sociedad de por sí empobrecida, se suma la llegada de un importante número de inmigrantes de origen europeo, provenientes en su mayoría de la Europa occidental, y en menor envergadura de Europa del este y el Cáucaso, que llegan al país escapando de persecuciones desde la primera guerra mundial y hasta fines de la segunda. No se trataba de poseedores/as de grandes fortunas que decidían aventurarse en nuevos horizontes, sino de sujetos cuya sobrevivencia amenazada les obliga a llegar, por lo general, con lo puesto, a arreglárselas en estas nuevas tierras.

En este período, la salud estaba a cargo de la propia población, la que se organizaba en las Sociedades de Socorro Mutuo. Eran momentos en que el Estado se debatía incansablemente en el cómo asumir el tema de la salud y la higiene, en especial a causa de la alta y permanente mortalidad infantil. "Es justamente en el curso de este proceso de crisis y cuestionamiento del modo de sumisión caritativa del régimen oligárquico, donde se levantará el problema histórico de la salud pública" (Illanes, 1993: 21).

\section{Tabla 2 MORTALIDAD DE LA POBLACIÓN}

\begin{tabular}{|c|c|c|}
\hline Edades & 1912 & 1913 \\
\hline Menores de 1 año & 38.836 & 30.135 \\
\hline 1 a 2 años & 8.136 & 9.368 \\
\hline 3 a 4 años & 3.760 & 3.706 \\
\hline 4 a 5 años & 1.345 & 2.772 \\
\hline 5 a 6 años & 1.146 & 1.355 \\
\hline 6 a 7 años & 859 & 1.057 \\
\hline
\end{tabular}

Fuente: Illanes (1993).

La alta mortalidad infantil fue el tema central de esta época. A la par de medidas para la construcción de la nación y en su modernización, estuvo la preocupación por terminar con este escenario. Sin embargo, sólo a fines de este período, en el gobierno de Alessandri, se tomó real conciencia de que la única forma de terminar con la desnutrición infantil era la implementación de un sistema de salud público, a cargo del Estado y no sólo de privados. Pero, además, se volvió imperativo poner fin -o por lo menos restar poder- a las Sociedades de Socorro Mutuo, las que se transformaron 
en importantes organizaciones de base, de reivindicaciones sociales y de una relativa independencia del "pueblo" con respecto al Estado.

Ahora bien, ¿cuáles eran las causas de la alta desnutrición, sobre todo infantil? Éstas pueden rastrarse en la importante migración rural-urbana, asociada a los pequeños espacios a los que accedían las familias en la ciudad, que las desvinculaba de la producción directa de la tierra, terminando con el abastecimiento agrícola familiar y dando lugar a cultivos muy reducidos, más asociados a las hierbas que a frutas y vegetales. Lo mismo sucede con el ganado y el acceso a las carnes, a lo que se suma la pobreza que impedía la compra de alimentos de todo tipo. De esta manera, se produjo una falta de aporte calórico-proteico. Pero no se trató sólo de la alimentación, sino también de las condiciones de vida donde la falta de elementos mínimos de salubridad, como agua potable y alcantarillado, produjo que los/as niños/as se enfermaran una y otra vez, sobre todo de fiebres y diarreas, perdiendo peso que no lograban volver a ganar.

En este contexto, el binomio madre-hijo/a va tomando fuerza, pero no por sí solo, sino inserto en un modelo mayor de familia ideal coherente con el modo de producción capitalista: la familia nuclear. En ella, los roles de mujeres y varones se encuentran claramente delimitados, recluyendo a las primeras al hogar, a lo doméstico y a la crianza, y a los varones a lo público, separándolos del cuidado de los/as hijos/as. Se definió, así, los aportes que cada género podía "naturalmente"4 entregar a la construcción de una nación fuerte e independiente, convirtiendo a las mujeres en las madres del país y dejándoles muy claro que su única tarea era ser buenas esposas, buenas madres y buenas mujeres.

Desde un inicio, las medidas contra la desnutrición infantil, fueran privadas o públicas, se basaron en el binomio madre-hijo/a y en la creencia, profundamente arraigada en Chile, de que el consumo de leche aseguraría el alcance de buenos índices nutricionales. En 1900 se crea el Patronato Nacional de la Infancia, institución privada dirigida por el Dr. Luis Calvo Mackenna y por Ismael Valdés Valdés, director del Hospital de Niños Manuel Arriarán. Se instalaron en barrios populares, poniendo en funcionamiento once "Gotas de Leche", dispensarios para la atención policlínica y la distribución de leche a las madres que, debido a su mal estado de salud (desnutrición, enfermedades de transmisión sexual -ETS- o tuberculosis), no alimentaban normalmente a sus bebés, peligrando la vida de éstos/as.

Pero no es hasta el gobierno liberal de Arturo Alessandri Palma (1920-1925) que la ciencia médica asume la necesidad de la lucha frontal contra la mortalidad infantil.

El término "naturalmente" se utiliza para dar cuenta de una visión esencialista de los roles de los géneros por parte de la sociedad de la época. Se usa entre comillas para señalar que la autora no está de acuerdo con esta visión. 
Ello responde, por una parte, a la necesidad de salvaguardar la mano de obra futura, pero también al recientemente incorporado modelo social, gracias al cual familia y hogar se transformaron en el núcleo fundamental de la sociedad.

Bajo este prisma, una vez detectados los focos de malnutrición infantil se dispuso de un ejército de salvación, capaz de llegar a las casas más pobres llevando la ayuda caritativa. Pero no fueron ni médicos, ni políticos, ni religiosos quienes iban a las poblaciones, sino el "Cuerpo de Señoras", distinguidas damas de la alta sociedad que, además de entregar los bienes enviados, enseñaban a estas otras madres, cuyos hijos/ as se desnutrían, cómo debían ejercer su principal y único aporte a la sociedad del momento: la maternidad.

"A la casa de Curicó nos visitan unas señoras ricas que vienen de Santiago y traen regalos, ropas y comida, a mi Paff para que me lo tome con leche y no me quede desnutrido. Son la tía Blanquita y la tía Martita. A las dos mi mamá las mira con mucho respeto y agradecimientos pero yo no les tengo gran cariño y sólo me admira que andan tan bien vestidas que parecen señoras ricas. A la Tegua le traen cintas para el pelo y le dicen la Chapecitos" (Hernández, Op. cit: s/p).

A fines del período de Arturo Alessandri Palma se fundan las leyes y normas que materializarán la visión de la medicina social. Es una idea en la que era fundamental la presencia de un sujeto capaz de relacionar la medicina con el hogar, y la ciencia con el humano, con lo cual nace la figura de la "visitadora social", el rostro humano de la ciencia y el Estado, alguien preparada y debidamente remunerada para "visitar" y "recorrer" los hogares del pueblo, reemplazando al Cuerpo de Señoras, pero siendo siempre mujeres.

De esta manera, lo que para aquellos tiempos se consideraba el "progreso en salud" se alinea con los mandatos de género hegemónicos, aportando su grano de arena al refuerzo de la idea y, más que a la idea, a la norma de que la crianza, los cuidados y, sobre todo, la nutrición son temas sólo de mujeres. De algún modo se consideran espacios "exclusivos y excluyentes", donde sólo ellas tienen cabida. Así, se pone en marcha un mecanismo de unión indisoluble entre mujer y reproducción, gracias al cual se les evalúa como "buenas" o "malas" mujeres según su calidad como madres. Es un respaldo, reconocimiento y fortalecimiento público y político de la mujer-madre, que será una constante a lo largo del siglo XX.

En 1927, el presidente Ibáñez, constando que uno de los grandes enemigos de la nutrición infantil eran las enfermedades venéreas pues impedían el correcto desarrollo fetal así como el amamantamiento de los/as bebés, organiza la sección de Higiene

Es una época en que el ser buena madre dependía de cuántos hijos/as paridos/as superaban los 6 ó 7 años de vida, es decir, cuántos no se morían de desnutrición. 
Social de la Dirección General de Sanidad, instalada en calle Artesanos, entregando atención médica y medicamentos gratuitos e iniciando la labor informativa, rompiendo así el tabú social que existía en Chile en torno al tema.

En 1937 entra en funciones la Ley de la Madre y el Niño, que extiende la distribución de leche a todos/as los/as menores de dos años, beneficio que se amplía con la creación del SNS en 1952, cuyo único objetivo durante sus primeros 50 años fue "mantener el óptimo estado nutricional de la embarazada para asegurar un desarrollo fetal armónico, una lactancia exitosa, y el desarrollo normal de la criatura" (MINSAL, 2002: 55).

La difusión del correcto cuidado de los/as hijos/as y del autocuidado de las embarazadas fue una cruzada estatal fundada en la creencia de que el máximo aporte femenino a la constitución de una nación moderna era ser "buenas madres". Potenciado por la industrialización, se fortalece el vínculo entre mujeres y mundo doméstico. "El Estado se convirtió en el garante de la protección jurídica, laboral y sanitaria de la maternidad como proyecto y de las madres como individuos" (Zárate, 2008: 129).

Sin embargo, y a pesar de todos los esfuerzos desplegados por organizar un modelo de salud pública efectivo, la década del '30 nuevamente se caracterizó por la crisis generalizada. "La mortalidad infantil en 1933 alcanzaba a 232 por mil nacidos vivos (...). La ciudad de Concepción tenía el triste privilegio de ser la ciudad con la más alta mortalidad infantil conocida en el mundo occidental: 328 por mil" (Illanes, Op. cit: 260). Por su parte, la tuberculosis y el tifus regaban muerte, sumándose a ello la falta de servicios básicos a lo largo del país.

\section{Institucionalización de la medicina social en Chile (Estado desarrollista y populista, 1938-1973)}

En 1938, la aparición del Frente Popular en el gobierno marca un antes y un después en el siglo XX, pasando de un Estado liberal a otro desarrollista y populista. Durante estos años se implementó una serie de medidas organizativas de la salud pública y del modo para enfrentar las pestes y enfermedades venéreas, medidas que por primera vez tuvieron efectos positivos.

Hacia 1939, bajo el gobierno de Pedro Aguirre Cerda, Salvador Allende se puso al frente del Ministerio de Salubridad, definiendo sus prioridades de acción claramente: la institución debía lograr, por una parte, organizar una "medicina social" en la que se considerara al pueblo no como objeto sino como sujeto; $y$, por otra, enfatizar su foco de atención en el binomio madre-hijo/a, único mecanismo para asegurar el cuidado de los/as niños/as, para lo cual creó, dentro del Ministerio, la Dirección Central de la Madre-Niño. Dos años más tarde incorporó a su lucha los problemas de la vivienda, argumentando que el hacinamiento y la insalubridad constituían causas importantes de la mortalidad infantil. Es así como en 1940 se aprobó el "Plan de Emergencia del Dr. Allende", la primera iniciativa nacional de saneamiento de las viviendas. 
Lamentablemente, para ese mismo año la crisis económica cundió en el país, dificultando la implementación de las ideas de la dupla Aguirre Cerda-Allende, pero este último, convencido de que en la leche estaba la respuesta a los problemas nutricionales, instaló los "bares lácteos". Fueron lugares que usaron la misma estética de las "chicherías" en las mismas zonas, donde se servía productos a base de leche, como sémolas y otros preparados, los que fueron abastecidos por la Central de Leche, gran industria del Estado. Fue tal su éxito, que al año siguiente la Central debió pedir ayuda a la Corporación de Fomento de la Producción (CORFO) ${ }^{6}$ para implementar más bares y para realizar estudios en fórmulas de suplementos lácteos. Fue el inicio de la industria nacional que hasta el día de hoy produce la enorme cantidad de alimentos, casi todos lácteos, que el MINSAL entrega gratuitamente en los consultorios de todo el país.

Ahora bien, las medidas implementadas recibieron un importante apoyo en 1946 con la creación de la Organización Mundial de la Salud (OMS). Además, en la década del '50 aparece un personaje clave en la historia de la superación de la desnutrición en Chile: Fernando Monckeberg ${ }^{7}$, quien en 1957 crea el Laboratorio de Investigaciones Pediátricas, uno de los precursores de lo que luego fue el Instituto de Nutrición y Tecnología Alimentaria (INTA). Desde aquella época, Monckeberg, junto a otros actores relevantes asociados a la Universidad de Chile, sostuvieron que la desnutrición no afectaba sólo a quienes morían, sino también a los/as que la sobrevivían, dañando de por vida su desarrollo y sus capacidades intelectuales, y provocando menores tallas. Desde esta visión, sus esfuerzos se centraron en que la desnutrición fuera reconocida como un problema político y no exclusivamente social, como la causa del subdesarrollo del país y no como una de sus consecuencias. Su argumento fue simple: una población dañada mentalmente es incapaz de llevar a su nación al desarrollo, reconocimiento para el cual tuvo que esperar la llegada de Allende al gobierno.

Desde el término del gobierno de Jorge Alessandri en los'60, el país vuelve a sumergirse en una crisis social importante: luego de un repunte, nuevamente la mortalidad infantil alcanza cifras que impactan, llegando a representar el 60\% del total de muertes. Esta situación se ve especialmente agravada por la continua llegada de familias migrantes a la ciudad, que deben enfrentarse a salarios bajos y a la escasez de viviendas. Estas familias terminan por aumentar los conventillos, con sus condiciones de higiene características, o por sumarse a tomas de terreno y poblaciones "callampa" desde la década del '50, que claramente no cuentan ni con alcantarillado ni con agua potable, agravando las condiciones de salud de los/as niños/as, así como su estado nutricional. 
En la década del'60 ingresan otros actores relevantes, como el Banco Mundial (BM) y el Fondo Monetario Internacional (FMI), que intentan potenciar el desarrollo de los países pobres a través de una ayuda económica que obliga a la implementación de sus políticas en el territorio nacional. De esta manera, el apoyo a la producción agrícola e industrial queda supeditado a la implantación del binomio madre-hijo/a como unidad objetivo para la supervivencia de la familia, reforzando los estereotipos de género ya presentes en nuestra sociedad. En general, se considera a las mujeres como sujetos pasivas del desarrollo, objetos de una política claramente caritativa, pues simplemente se entregan ciertos bienes asumiendo que ellas los repartirán entre toda la familia. De este modo, se logra mantener a las mujeres en sus hogares sin entregarles ninguna herramienta que les permita empoderarse en tanto sujetos de derechos.

Siguiendo esta visión, las instituciones líderes del desarrollo, y en especial EE.UU., ven a las mujeres latinoamericanas como las causantes del "desfase entre crecimiento económico y demográfico", la razón de la pobreza. En otras palabras, el problema es que los/as pobres tienen demasiados/as hijos/as, a los que no pueden mantener, por lo cual EE.UU incluye las políticas de natalidad en sus implementaciones prioritarias, planteándolas como responsabilidad exclusiva de las mujeres, las madres de la sociedad. En el caso particular de Chile, la decisión de poner en marcha la planificación familiar responde también a la necesidad de disminuir los altos índices de mortalidad materna por aborto.

En 1970, Allende asume como el nuevo presidente de Chile, inaugurando una estrecha colaboración entre el poder político y las propuestas de Monckeberg. Es durante el gobierno de la Unidad Popular, entonces, que se inician los tiempos de las medidas más importantes para la superación de la desnutrición, siguiendo cuatro líneas principales: i) la distribución de leche para la población en riesgo (embarazadas, madres en período de lactancia, bebés y niños/as); ii) aumento de infraestructura para el ejercicio de la salud pública, en especial de los consultorios, donde se potencia la educación de las mujeres-madres; iii) impulso a la educación y a la distribución de alimentos en los establecimientos educacionales; y iv) saneamiento asegurando agua potable y alcantarillado.

Se instaura el Plan de Emergencia en Salud, que significó medio litro de leche por niño/a al día, además de un plan especial contra las diarreas infantiles. A ello se sumó el Plan de Leche o su repartición gratuita a todos/as los/as menores de 2 años, a preescolares, a mujeres embarazadas y madres en período intergestacional, mientras que los/as escolares estaban cubiertos/as a través de la Junta Nacional de Auxilio Escolar. Para la sociedad en general, se dictó el decreto de pasteurización de la leche. Se realizaron campañas de acción masiva, en las cuales profesionales de la salud acudían a las bases para solucionar sus problemas médicos, además de capacitar a las mismas pobladoras en trastornos que podían ser solucionados en los hogares, liberando en gran medida los centros asistenciales. 
Pero una de las medidas más importantes de Allende, y que tuvo mayor impacto en la malnutrición en Chile, fue la incorporación oficial de la mujer a la salud bajo la figura de "responsable de salud". Bajo esta óptica, se reforzaron los CEMAs ${ }^{8}$ creados entre 1965 y 1969, agrupaciones populares de base que se convirtieron en verdaderas escuelas para ser buenas mujeres, buenas madres. Con esto, el presidente terminó de sellar el proceso iniciado desde principios de siglo de asociación indisoluble entre los cuidados, la salud y las mujeres, legitimando por completo la ausencia de los varones no sólo del cuidado de los/as otros/as miembros de su familia, sino también de sí mismos. Hoy, uno de los principales problemas de la salud pública son los factores de riesgo en el estado de bienestar de los hombres, pues éstos no acuden a los centros de salud, niegan las enfermedades y, en general, no se responsabilizan por los cuidados necesarios de un/a bebé. Estereotipos o géneros hegemónicos que no son gratuitos, sino que han sido construidos y legitimados a lo largo del siglo XX desde variados ámbitos de la vida social. La salud pública ha contribuido desde dos brazos de la medicina social: el consultorio y la publicidad del Ministerio.

\section{Superación definitiva de la desnutrición (Dictadura, 1973-1989)}

La irrupción de la dictadura en Chile dio curso a importantes quiebres y giros en la vida social, política y económica de la nación, en especial a partir de los ochenta, cuando se sentaron las bases de la instauración profunda de la modernidad y la capitalización del país.

En esta década se materializó una importante crisis económica que venía siendo gestada desde los '70, llegando a llamarse esta época la década perdida del desarrollo, transcurrida en un contexto en que el "primer mundo" se alineaba en la necesidad de implementar lo que hoy conocemos como capitalismo y libre mercado en aquellos países cuyo desarrollo debía ser impulsado. En Chile, esto fue vivido desde dos grandes bloques: la entrada en escena de los "Chicago Boys" y la exigencia de las instituciones líderes del desarrollo (BM y FMI) para entregar su ayuda económica. De esta manera, la época de modernización y capitalización más aguda comenzó en la década del '80, cuando se aplicaron normas de libre mercado, por una parte, disminuyendo el tamaño del Estado y del gasto social y, por otra, aumentando enormemente la privatización en la salud (Isapres), en la educación (Universidades privadas) y en la protección social (AFP), además de las grandes empresas del Estado.

Todas estas medidas significaron cambios profundos en la vida cotidiana, trastrocando valores, creencias, la moral y la ética, y la estructura simbólica de nuestra forma de vida. Se aceleraron sus ritmos, teniendo cada vez menos espacio para la alimentación, privilegiando el trabajo, así como las tecnologías que ahorran tiempo dedicado 
a la reproducción. Se acentuó una identidad católica y militar chilena pero, por sobre todo, se impulsó el proceso de individuación e individualismo. Y a pesar de todos estos importantes cambios, las políticas nutricionales con sus concepciones de género se mantuvieron, reforzándolas aún más.

Los '70 y '80 son las dos últimas décadas de la desnutrición en Chile, debido, principalmente, a las estrategias implementadas por Allende y Monckeberg, a las que éste último dio continuidad durante la dictadura gracias al apoyo del general Gustavo Leigh.

\section{Tabla 3} AMÉRICA LATINA:TASAS DE MORTALIDAD INFANTIL 1969-1980 (MUERTES DE MENORES DE 1 AÑO POR MIL NACIDOS VIVOS)

\begin{tabular}{|l|c|c|c|c|c|}
\hline & 1960 & 1965 & 1970 & 1975 & 1980 \\
\hline Argentina & 62.4 & 56.9 & 58.8 & $44.6 \mathrm{a}$ & $40.8 \mathrm{~b}$ \\
\hline Colombia & 99.8 & 82.4 & 70.4 & 55.0 & \\
\hline Costa Rica & 68.6 & 69.3 & 61.5 & 37.1 & $22.9 \mathrm{c}$ \\
\hline Cuba & 35.4 & 38.4 & 38.3 & 27.3 & $19.1 \mathrm{c}$ \\
\hline Chile & 120.3 & 95.4 & 79.3 & 55.4 & 31.8 \\
\hline Ecuador & 100.0 & 93.0 & 76.6 & $57.5 \mathrm{a}$ & $64.4 \mathrm{~b}$ \\
\hline Perú & 92.1 & 74.0 & 65.1 & 53.8 & 50.5 \\
\hline Paraguay & 90.7 & 83.6 & 93.8 & 84.9 & $91.4 \mathrm{~b}$ \\
\hline Uruguay & 47.4 & 49.6 & 42.6 & 48.6 & 37.4 \\
\hline Venezuela & 52.9 & 46.4 & 49.2 & 43.7 & 31.8 \\
\hline
\end{tabular}

a: año 1977

b: año 1978

c: año 1979

Fuente: Illanes (1993).

Como aún avanzados los '70 había amplias zonas donde no existían los consultorios, hubo que desarrollar un programa enorme de habilitación de centros de salud, lo que implicó extender los programas de planificación familiar, vacunación y control de niño sano. Dentro de este aparataje, fue la entrega de leche gratuita en los consultorios lo que aseguró que las madres efectivamente acudieran a los centros de salud, donde se desarrolló toda la labor de promoción, es decir, la educación de las madres. Debido a esto hay una fuerte relación entre la cantidad distribuida de alimentos y el número de consultas y controles realizados a la madre y los/as niños/as.

En 1974 el Servicio Nacional de Salud contó con su propia marca de leche, la famosa Purita. Luego, en 1975, Monckeberg fundó la Corporación para la Nutrición Infantil (CONIN) y trabajó para que la entrega de Purita se extendiera hasta los 2 años de edad, además de impulsar la fabricación de alimentos proteicos para niños/as mayores y la creación de un sistema de vigilancia nutricional, cada tres meses, para los/as meno- 
res/as de 6 años. En 1987, el Programa Nacional de Alimentación Complementaria9 (PNAC) fue establecido por ley como beneficio universal para niños/as menores de 6 años y embarazadas.

Tabla 4

\begin{tabular}{|c|c|c|}
\hline Año & Kilogramos distribuidos & Índice \\
\hline 1970 & 12.695 .368 & 100,0 \\
\hline 1971 & 19.548 .162 & 154,0 \\
\hline 1972 & 20.064 .644 & 158,0 \\
\hline 1973 & 21.292 .847 & 167,7 \\
\hline 1974 & 23.982 .507 & 188,9 \\
\hline 1975 & 27.399 .430 & 215,8 \\
\hline 1976 & $30.146 .770 a$ & 237,5 \\
\hline 1977 & $36.913 .270 a$ & 290,8 \\
\hline 1978 & 29.826 .536 & 234,9 \\
\hline 1979 & 28.718 .760 & 226,2 \\
\hline 1980 & 29.214 .871 & 230,1 \\
\hline 1981 & 29.782 .354 & 234,6 \\
\hline 1982 & 30.287 .061 & 238,6 \\
\hline
\end{tabular}

Fuente: Illanes (1993).

Todas estas medidas fueron supervisadas y acompañadas por la mirada vigilante del INTA, que nace en el marco de la reforma de los '70 de la Universidad de Chile a cargo de Monckeberg. Éste reunió la dirigencia de las dos instituciones nutricionales más importantes de la época, el INTA y la CONIN, a lo que se suma el Consejo Nacional para la Alimentación y Nutrición (CONPAN) del gobierno. De esta manera, fue Monckeberg quien organizó legalmente las acciones de nutrición y alimentación, basadas en el Sistema de Alimentación y Nutrición para la recuperación y prevención de la desnutrición grave.

La continuación de las líneas implementadas en el gobierno de la Unidad Popular en cuanto a desnutrición y mortalidad infantil, así como el prestigio de Monckeberg, fue lo que permitió terminar de superar estos importantes problemas de malnutrición al llegar a la década del '90, pues reunieron en las políticas públicas lo sanitario con lo nutricional. Pero esta estrategia no incluía sólo asegurar el alcantarillado y el consumo de leche y alimentos fortificados en niños/as y embarazadas, también la asignación total de las responsabilidades reproductivas a las mujeres. Así, por ejemplo, una vez que se diagnosticaba un/a niño/a desnutrido/a, éste/a se le quitaba a la familia y era 
llevado/a al CONIN que le correspondía, donde, además de recuperar su peso, la madre debía asistir a reuniones con las cuidadoras para aprender a ser una "buena madre", una "buena mujer". Sólo con esta condición el/la niño/a era devuelto/a a su hogar.

"Por ejemplo, los CONIN fueron centros desarrollados para atender a los niños desnutridos, el doctor Monckeberg era el gran dueño de estos centros que eran privados, pero pagados por el Estado. Ese niño prácticamente se le quitaba a la madre y se enviaba a este centro y no se devolvía hasta que se consideraba que la madre estaba en condiciones de recibirlo nuevamente. Para esto, esta madre iba a este centro o tenían un sistema de cuidadoras que eran señoras que cuidaban varios niños, que se les pagaba por eso, y las otras madres iban a aprender cómo cuidar a los niños. Una vez que estaban recuperados nutricionalmente y la madre estaba certificada, se le devolvía. Es por eso que el estigma de la desnutrición es algo tan fuerte porque en el fondo es un estigma a la madre. Tú habías fallado en tu rol de madre al tener un hijo desnutrido" (Marcela Romo, Departamento de Nutrición, MINSAL).

A la vez, desde el personal médico se instauró en la población la visión de que un/a niño/a con un kilo de sobrepeso era sano/a, instalándose en el imaginario la homología entre salud y caritas redondas, rosadas y sonrientes. Para esta estrategia fue fundamental la educación, promoción y publicidad de la salud a través de su sistema público, sobre todo de los consultorios. En un contexto en que la industria alimentaria no se desarrollaba aún a niveles en que los productos fueran accesibles para todos/ as y en que los ingresos económicos no permitían aún, a la mayoría de la población, la elección de sus alimentos, esta asociación entre salud y gordura fue necesaria para lograr llegar a pesos adecuados, pues logró establecer una imagen visual de a dónde se quería llegar. Esto, por supuesto, en un escenario en que los alimentos disponibles tenían, en su mayoría, un contenido graso y de azúcares bastante moderado. Se definió así una ideología de lo saludable que estuvo muy marcada por el consumo regular de leche, en especial en mujeres y niños/as, y por el "más es mejor".

Ahora bien, esta ideología de lo saludable necesitó de un actor clave que estuviera a su cargo, vale decir, alguien a quien responsabilizar asegurando por alguna vía que se cumpliera, acomodándose a otro tipo de ideología, la de género. Como sabemos, desde mediados de este mismo siglo en adelante las mujeres abrimos importantes espacios, asegurando nuestro derecho a voto, a la educación y al trabajo digno. Sin embargo, el campo que se ha tratado en este artículo (aquél de la reproducción y el cuidado de los/as hijos/as) se erige como uno de los núcleos más duros de la concepción patriarcal de los roles de género. Hay un mensaje del último siglo que ha calado profundamente en las mujeres, ingresando en su universo simbólico, lo que puede verse, por ejemplo, en la necesidad de las anfitrionas de atiborrar con comida a quienes las visitan. Aquí jugaron un importantísimo rol las políticas públicas, pues no se trata sólo de la memoria de la carencia sino también de una profunda asociación entre la comida y el cariño, entre la mujer y la madre. 
Frases como "los/as niños/as son los/as adultos/as del futuro" o "los/as niños/as son el futuro de Chile" se volvieron preceptos importantísimos a la hora de asegurar la continuidad de la nación chilena. Es un contexto en el cual los dos grandes actores fueron las mujeres-madres y las políticas públicas nutricionales-sanitarias, formando dos dúos inseparables.

\section{MODERNIZACIÓN NEOLIBERAL, EXPANSIÓN ECONÓMICA Y LUCHA CONTRA LA OBESIDAD (1990-2009)}

\section{La aparición de la obesidad (1990-2000)}

En la década de los '90, finalmente se soluciona la desnutrición, arrastrada desde inicios del siglo XX, gracias a las duplas mujer-madre y políticas públicas nutricionalessanitarias. Pero, además, en el país vuelve la democracia, iniciando el largo reinado de la Democracia Cristiana (DC $)^{10}$ en el gobierno, la que, contrario a lo que se podría haber esperado, profundiza aún más el modelo neoliberal en nuestra sociedad. A partir de los noventa se supera la crisis económica de la década anterior, iniciando un período de bonanza con el consecuente incremento de la capacidad adquisitiva de la población, el que viene acompañado de la liberalización total de los mercados y la apertura a la industria global, incluida, por supuesto, la alimentaria. Comienza el boom de las golosinas, la aparición de productos con mucho azúcar, ricos en grasas y, sobre todo, alimentos procesados a los que la población no había tenido acceso anteriormente.

Básicamente, en la obesidad ha influido el aumento del ingreso, lo que otorgó a los/as niños/as, por primera vez, la posibilidad de elegir en un escenario en que los alimentos más calóricos son efectivamente los más sabrosos, pues si bien la grasa o el aceite no tienen sabor por sí mismos, realzan el gusto propio del alimento (un huevo duro no tiene el mismo sabor que un huevo frito). Recordando la tabla de transición nutricional presentada en un inicio (tabla 1), el país supera una primera etapa pre transicional para ingresar de lleno en el momento de la transición.

En esta época, el panorama de las clases bajas en el país se compone de hijos/ as desnutridos/as con madres obesas, mientras que en las clases medias comienza a aumentar preocupantemente la obesidad en los/as adultos/as. Pero a pesar de la aparición de la obesidad en el escenario y de que las cifras de desnutrición se superaran enormemente, las duplas mujer-madre y políticas nutricionales-sanitarias siguen en pie, aplicando un paquete de medidas orientadas a aumentar el peso a un contexto de estado nutricional que ya no lo requiere. En el fondo, al país le lleva una década entera darse cuenta de que el escenario ha cambiado y que el tipo de malnutrición al que debe enfrentarse se relaciona mucho más con la calidad de los alimentos que

Partido político de centro-izquierda, miembro de la coalición denominada Concertación de Partidos por la Democracia. 
con su escasez. Si antes una de las mayores preocupaciones estaba dada por la falta, a partir de esta época es algo que deja de inquietar, teniendo que lidiar con lo contrario, la sobreabundancia.

Las políticas que erradicaron la desnutrición en nuestro país tuvieron tal eco, se asentaron tan profundamente en nuestros imaginarios, que hasta la actualidad son difíciles de readecuar a los nuevos contextos de estado nutricional. Es necesario considerar que se instaló todo un sistema para asegurar la leche, un alimento sumamente calórico-proteico, a todos/as los/as niños/as de Chile pertenecientes al sistema público, ya sea a través del PNAC o de la Junta Nacional de Auxilio Escolar y Becas (JUNAEB). A lo anterior, se suma toda una cultura que reconoce como saludable al bebé y al infante "un poquito pasadito de peso", y a las mujeres con niños/as sanos/as como las buenas, las ideales. De esta manera, si bien la obesidad aparece desde la década de 1990, la reacción sanitaria y social para controlarla se ha visto dificultada por la herencia de la lucha contra la malnutrición por carencia.

"Las abuelas, si el niño está normal te dicen 'está desnutrido,' 'no está comiendo mucho,' y si el niño no come por algunas horas hay una sensación de angustia de algunas familias. Eso repercute. La pediatría de los '60, '70, hablaba de que el niño con un kilo más era el que sobrevivía a las 4 neumonías y las 4 diarreas que tenían en los primeros 4 años de vida. Hay un concepto ahí muy fuerte desde la salud que también entra en sintonía con este modelo" (Tito Pizarro, Agencia de Inocuidad de Alimentos).

\section{El asentamiento de la obesidad}

Iniciando el siglo XXI se vuelve imposible continuar negando lo que las cifras delatan por sí mismas: la obesidad ha llegado para quedarse y se ha transformado en un problema con carácter de epidemia, pues se multiplica a pasos agigantados. No se trata sólo de cambios en las conductas alimentarias sino también en la oferta de los alimentos ricos en grasas, azúcares y calorías.

"El mejoramiento económico ha significado cambiar el estilo de alimentación hacia una dieta caracterizada por alto consumo de alimentos procesados, con comida rápida rica en grasas saturadas y altamente calóricas. El consumo de grasas ha aumentado de 13,9 kg/persona/año en 1975 a 16,7 kg/persona/año en 1995. Las tendencias del consumo nacional muestran un importante aumento en el consumo de carne, principalmente cerdo y pollo, cecinas, productos lácteos, y una disminución en el consumo de pescados, frutas, verduras, cereales y leguminosas" (MINSAL, Op. cit: 73).

Este escenario se acompaña de un importante desarrollo de la industria alimentaria que, por lo menos en Chile, asocia los bajos precios a los productos con más grasas y calorías, y los más caros a los más saludables. Se genera, además, una importante 
brecha socioeconómica en la situación nutricional nacional, estando la obesidad concentrada en las clases bajas y la preocupación por el cuidado del cuerpo en las altas.

A esto se suma una vida cada vez más sedentaria, en la que juega un rol relevante la urbanización de los últimos cincuenta años. El rápido desarrollo de las tecnologías afecta tanto la producción como las distintas formas de recreación, en las que la oferta televisiva, los juegos tipo Nintendo y las computadoras reemplazan velozmente las distracciones al aire libre y con actividad física.

"Hoy somos mucho más sedentarios de lo que siempre fuimos. Esto tiene que ver con las comunicaciones, con la urbanización, con los elementos de comodidad que te permite la modernidad, la televisión, el auto, una serie de elementos que te hacen estar sentado. Hoy ni siquiera los trabajos de mayor intensidad como son la minería, la construcción o la ganadería, todo se hace hoy con máquinas, entonces hay un elemento que es más determinante incluso que la alimentación misma, que tiene que ver con lo sedentarios que nos pusimos los chilenos" (Tito Pizarro, Agencia Chilena de Inocuidad de Alimentos).

Cuando se creó el Servicio Nacional de Salud en 1952, se definió la superación de la desnutrición como meta sanitaria para los próximos cincuenta años, es decir, hasta el año 2002. Esto obligó al MINSAL a iniciar actividades de evaluación y redefinición de sus objetivos estratégicos el año 2000, cuando se realizó la Primera Encuesta Nacional de Calidad de Vida, que incluyó preguntas referidas tanto a los hábitos alimenticios como a la percepción del propio peso. Los datos arrojados obligaron a la institución a abrir los ojos frente a la obesidad, asumiendo que, según la OMS, ha alcanzado dimensiones de epidemia mundial con más de mil millones de individuos con sobrepeso, de los/as cuales al menos 300 millones son obesos/as.

De esta manera, desde el año 2002 -y hasta 2010- el nuevo objetivo del MINSAL es contribuir a la reducción de la obesidad y de la prevalencia de las enfermedades crónicas no transmisibles (o ECNT), en especial hasta la edad escolar. Lamentablemente, su disminución para el año 2007 es casi nula, volviendo difícil el cumplimiento de las metas establecidas. No obstante, hay que considerar que aunque los nuevos objetivos estratégicos son publicados en 2002, no es hasta el año 2005 -y en especial 2006- que se comienza a implementar las medidas más importantes en contra de la malnutrición por exceso.

El MINSAL es un ministerio que se ha fortalecido enormemente en los últimos sesenta años, sobre todo su Departamento de Nutrición, llegando a ejercer una importante influencia sobre la población. Actualmente, la institución se plantea una distinción clara entre las intervenciones estructurales y las soluciones individuales que pueden ofrecerse. Las primeras se refieren a políticas capaces de llegar a la población en su conjunto sin distinción de clase, edad, género o raza, como por ejemplo, una vez detectada una alta prevalencia de enfermedades causadas por falta de yodo, la respuesta estructural es yodar un alimento muy usado por todos/as: la sal. Lo mismo 
con el ácido fólico en las harinas o con el flúor en el agua. Ahora bien, este tipo de decisiones son tomadas por el MINSAL y son impuestas a la población. Vemos que, de alguna manera, se trata de medidas bastante paternalistas, en las cuales la institución es quien decide por las personas a las cuales representa, argumentando que es más barato, rápido y eficiente implantar este tipo de normas antes que educar a toda la población. Una de las medidas más recientes en esta línea es la prohibición de las grasas trans en las margarinas.

Por otra parte, el Ministerio se preocupa de implementar políticas individuales que se refieren, básicamente, a la garantía de cobertura de enfermedades cuya causa ya ha sido atacada estructuralmente, haciéndose cargo sólo de las excepciones; y también de la promoción en salud. Esta última abarca distintas estrategias, siendo una de las principales la educación entregada en los centros asistenciales durante los controles de niño/a sano/a, así como la campaña publicitaria iniciada por el MINSAL en los últimos tiempos, a través de comerciales transmitidos por televisión y del desarrollo de una importante folletería.

En 2005 se implementó en todo el país la Estrategia de Intervención Nutricional a través del Ciclo Vital para la Prevención de la Malnutrición por Exceso y otras ECNT. En ella se establece una serie de actividades basadas en la consejería en vida sana, dirigidas en una primera etapa a las embarazadas y niños/as menores de 6 años. Luego, en 2006, se lanza la Estrategia Global contra la Obesidad (EGO Chile), que se inserta en la Estrategia Global sobre Alimentación Saludable, Actividad Física y Salud, de la OMS y la Organización Panamericana de Salud (OPS). Su meta fundamental es, por supuesto, disminuir la prevalencia de la obesidad, para lo cual involucra a treinta y tres organismos públicos y privados, entre los que se cuentan los Ministerios de Salud, Agricultura y Educación, aunando los esfuerzos de todos los sectores: sanitario, familiar, comunitario, escolar, empresarial y académico.

EGO tiene como pilares fundamentales la actividad física y la alimentación, de modo que se propone: i) educar a la población respecto a qué es una dieta saludable y a la importancia de la actividad física, principalmente, a través de los controles habituales y la distribución de la Guía para una Vida Saludable; ii) capacitar al equipo de salud en la aplicación del modelo de intervención nutricional a través del ciclo vital; iii) incorporar nuevos controles de salud; iv) vigilar el etiquetado obligatorio de los alimentos y apoyar a la población para que aprenda a leerlos correctamente; y v) acrecentar los esfuerzos para generalizar la lactancia materna hasta los seis meses.

Lo que se menciona como Guía para la Vida Saludable se refiere a lo que el MINSAL define como una alimentación y conducta sana, lo que incluye consumir 5 porciones de frutas y verduras de distintos colores todos los días, reduciendo con ello la incidencia del cáncer, enfermedades del corazón, diabetes y ayudando en el control del peso. También, consumir de 6 a 8 vasos de agua al día y tres veces productos lácteos; legumbres y pescado dos veces por semana y evitar las grasas saturadas, el colesterol, 
el azúcar y la sal (para lo cual enfatiza en la importancia de leer las etiquetas nutricionales). Por último, caminar mínimo 30 minutos al día, ejercitándose 30 minutos tres veces por semana, hacer estiramientos e incorporar pausas en el trabajo que estimulen los recreos activos.

Ahora bien, ¿qué sucede con los discursos nutricionales de la población en este contexto? ¿Sigue siendo la leche un producto fundamental en la alimentación chilena? ¿Sigue siendo el sobrepeso el ideal de un cuerpo sano? ¿Somos aún las mujeres encargadas del cuidado y nutrición de las familias, en especial de los/as niños/as?

Claramente, el ingreso al siglo XXI ha significado una diferencia importante en la historia nutricional chilena, marcando su entrada en la etapa post transición con una fuerte incidencia de la malnutrición por exceso. Y, aunque podríamos comparar esta fase, por oposición, con la primera y la prevalencia de la desnutrición, existe una diferencia fundamental entre ellas: el fuerte desarrollo experimentado por el MINSAL desde 1952 y la importante credibilidad que ha adquirido frente a la población. De esta manera, a la hora de enfrentar la obesidad el país ya cuenta con los mecanismos necesarios, como lo son una infraestructura que permite la cobertura total y, por ende, la promoción de la salud y la fuerza política suficiente.

A los programas ya mencionados se suma el Programa de Alimentación Complementaria, que comienza a ser afinado por el Ministerio (y que sigue operando en nuestro país), además de la implementación de tratamientos específicos contra la obesidad en los servicios de salud. Estos últimos, si bien resultan muy caros, son también bastante efectivos. Una vez que la institucionalidad reacciona al cambio de la situación nutricional del país, pone en funcionamiento todo su arsenal para cambiar el ideal de un cuerpo sano, para incentivar a la población a tener una alimentación equilibrada y para combatir el sedentarismo.

En este sentido, es impresionante cómo los mandatos del MINSAL calan en los individuos. No nos referimos a los comportamientos efectivos, pero sí a los discursos que las personas sostienen sobre lo bueno y lo malo para comer. Chile es un país donde, si bien las conductas alimenticias pueden variar enormemente-sobre todo por clase-, las ideas e ideologías nutricionales son bastante compartidas, como lo muestran los testimonios a continuación:

"Es súper bueno comer frutas y verduras; carne, como un poco, es que en el colegio también estaba la nutricionista del comer moderado, equilibrado, no en exceso, no en la noche" (Carolina, 30 años, Ñuñoa).

"Procuro comer bastante verdura, no comer puro carbohidrato, no comer puro pan, comer de vez en cuando legumbres. Tengo la idea de que tengo que comerverduras, tampoco es algo tan consciente" (Paula, 25 años, Providencia). 
"El menú era, qué sé yo, un día tallarines, otro día legumbres, otro día pescado, cuando querían comer. Pero en general, sí, un día cada cosa. Siempre se ha comido mucha ensalada en la casa y los platos de fondo siempre han sido platos comunes. En el fondo, dándoles en el gusto y que sea una comida relativamente equilibrada" (Tamara, 58 años, Ñuñoa).

"Más o menos tradicional, tratando de que fuera con verduras, con frutas, con lácteos, todo lo más, lo más tradicional. Siempre hemos privilegiado la comida casera" (Verónica, 52 años, Santiago Centro).

En general, la población comparte la idea de que una alimentación sana es equilibrada, que hay que comer de todo pero sin excesos, que es necesario consumir mucha fruta y verdura y legumbres por lo menos una vez por semana. Es decir, un calce perfecto con los mandatos que el MINSAL ha estado reforzando en los últimos tres años. Se trata de una ideología en la cual los lácteos siguen ocupando un lugar central. No sólo todos/as conocemos perfectamente la campaña publicitaria de TV del Tómate la leche, sino que también nos escandalizamos cuando somos testigos de niños/as cuya alimentación no incluye este tipo de producto. Incluso en un contexto mundial en que los cuestionamientos de la pertinencia del consumo de lácteos en personas adultas toman cada vez más fuerza, somos uno de los países en que se consume más leche, en todas las edades.

En cuanto a causas de la obesidad, a las ya expuestas se suma la composición nutricional familiar como un importante factor. Así, en núcleos donde los padres o abuelos/as (las personas más cercanas a los/as niños/as) son obesos/as aumenta enormemente la probabilidad de que quienes estén en desarrollo imiten el mismo tipo de comportamiento. En este sentido, llama la atención que los programas implementados por el MINSAL continúen dejando fuera a los varones adultos y centrándose en las embarazadas y los/as niños/as, exclusión que enfatiza la idea de que las políticas públicas nutricionales se basan, aún, en un modelo de género en que se atribuye a las mujeres la responsabilidad total del cuidado y alimentación de los/as hijos/as, reforzando todavía la homologación de la mujer con la madre.

\section{A MODO DE CONCLUSIÓN}

La constitución de la medicina social en Chile se realizó con fuerza a lo largo del siglo XX, abocándose a la superación de la malnutrición por carencia. La nutrición se constituyó en un ámbito de la historia social chilena que, a diferencia de otros, fue reforzada por la sucesión de los distintos enfoques de Estado, lo que puede deberse a que los gobiernos se sirvieron de ella para legitimarse frente a una población con graves problemas sanitarios. Una de sus consecuencias más visibles fue, como ya se ha señalado, la importante credibilidad con que cuenta el MINSAL en nuestro país, beneplácito con el que no cuenta la mayoría de nuestros Ministerios. A ello se debe sumar que, tradicionalmente, la salud se ha amparado en criterios científicos para establecer sus verdades y creencias, lo que ha ahuyentado las dudas de la población en 
general, sobre sus medidas y principios. Y, si bien la ciencia comienza a ser puesta en duda, la medicina oficial y, sobre todo, los temas nutricionales han sabido ampararse en nuevas configuraciones político-sociales, como son las alianzas entre salud y belleza. De esta manera, se puede situar al MINSAL como un espacio relevante de poder político, cuya injerencia social ha sido, lamentablemente, escasamente problematizada desde las ciencias sociales.

Desde el siglo XX, las políticas nutricionales han configurado un imaginario de cuerpo ideal: aquella constitución física que da cuenta de un/a sujeto saludable, un/a ciudadano/a capaz de cumplir con sus responsabilidades y derechos, por ende, al guien que está dentro de la norma. Durante mucho tiempo se trató de cuerpos "gorditos", "rellenitos", "rosaditos", en pos de lograr combatir la desnutrición y proteger a los/as infantes de la cantidad de enfermedades que los/as atacaban en sus primeros años de vida. Pero, hoy en día, esa constitución inicial intenta ser reemplazada por cuerpos atléticos, estilizados, que den cuenta de una batalla ganada contra el enemigo principal: las grasas. Hoy, para alcanzar la norma y el estatus que da el estar a la "altura", se debe ocupar un físico delgado y fibroso. No por casualidad en los últimos años somos testigos del surgimiento de deportistas olímpicos como modelos, cuando anteriormente eran vistos como cuerpos extraños, deformados por el ejercicio, sobre todo las mujeres.

Desde el siglo XX también, las políticas públicas sobre nutrición han construido un imaginario social sobre el deber ser femenino, sobre lo que es ser mujer. Así como nos decía Simone de Beauvoir, "la mujer no nace, se hace", y en base a las directrices públicas nutricionales hay una serie de pasos a cumplir para llegar a serlo. Hay que ser madre y, una vez madre, asumir el protagonismo en el cuidado de los/as cercanos/as. Incluso, responsabilizarse si es que dicho cuidado no da los efectos deseados. Desde 1900 el Estado ha reforzado la idea de que mujer-madre es quien cuida, quien nutre, quien entrega cariño, quien canaliza sus afectos por medio de los alimentos. Desde 1900 el Estado ha reforzado una construcción de género donde lo femenino aparece íntimamente relacionado con lo doméstico, delegando las responsabilidades de cuidado en las mujeres.

En la génesis histórica de las políticas públicas alimentarias pudimos ver cómo el discurso oficial ha ido entretejiendo una trama en que lo nutricional ha sido situado en lo doméstico, a la vez que se le ha otorgado un cuerpo responsable, generizándolo en lo femenino. De hecho, a través de sus leyes y programas, la oficialidad ha designado a las mujeres como las responsables de este espacio y, por ende, también del estado nutricional de sus integrantes, del cuidado de los/as otros/as, de asegurar la reproducción. Es importante recordar que, a pesar de los cambios en las necesidades alimentarias de la población y del aumento de la evidencia de que es fundamental la inclusión de los varones para prevenir las enfermedades por malnutrición, las políticas públicas continúan excluyéndolos y centrándose exclusivamente en las mujeres. 


\section{BIBLIOGRAFÍA}

Albala, Cecilia et al. (2000): Obesidad: un tema pendiente. Santiago de Chile: Editorial Universitaria.

Hernández, Guillermo (2008): Historia de la familia Hernández. Santiago de Chile: Texto no publicado.

Illanes, María Angélica (1993): En el nombre del Pueblo, del Estado y de la Ciencia. Historia social de la salud pública, Chile 1890-1973." Santiago de Chile: Colectivo Atención Primaria.

MINSAL (2002): Construyendo una política pública en salud. Plan AUGE: Una base sanitaria para la Reforma del Sistema de Salud Chileno, Documento de Trabajo para los talleres "Planificando el futuro de la salud". Santiago de Chile: Ministerio de Salud.

Zárate, María Soledad (2008): "Las madres obreras y el Estado chileno. La Caja del Seguro Obligatorio, 1900-1950", en Montecino, Sonia (comp.): Mujeres chilenas, fragmentos de una historia. Santiago de Chile: Catalonia. 